\title{
Reply to a letter to the editor: Diabetic Retinal Pigment Epitheliopathy: Fundus Autofluorescence and Spectral-Domain Optical Coherence Tomography Findings
}

\author{
Eui Chun Kang ${ }^{1} \cdot$ Yuri Seo $^{1} \cdot$ Suk Ho Byeon ${ }^{1}$
}

Received: 12 May 2016 / Accepted: 23 May 2016/Published online: 24 June 2016

(C) Springer-Verlag Berlin Heidelberg 2016

We thank Uzun et al. for their valuable comments on our article [1]. We reported that eyes with diabetic retinal pigment epitheliopathy (DRPE) showed decreased visual acuity, thinning of the outer retina, and thickened choroid than did eyes with proliferative diabetic retinopathy (PDR). Choroidal thickness (CT), which was one of the clinical parameters evaluated in our study, could be influenced by many factors, as Uzun et al. have pointed out [2]. As our study was limited by its retrospective design, local/systemic and environmental factors affecting $\mathrm{CT}$ could not be evaluated owing to limited data availability. Axial length is highly correlated with the spherical equivalent [3]; however, we did not evaluate the axial lengths in the DRPE and PDR groups. As we mentioned in the Results section of the manuscript, there were no differences in the baseline characteristics, including the spherical equivalent, presence of hypertension, and insulin use, between the DRPE and PDR groups. Therefore, we speculated that the axial lengths would not be different between the groups. Moreover, the body mass index values were not statistically different between the DRPE and PDR groups $(21.92 \pm 1.45$ vs. $\left.21.79 \pm 2.59 \mathrm{~kg} / \mathrm{m}^{2}, P=0.902\right)$; however, this result was not presented in our manuscript.

To minimize the effect of diurnal variation in $\mathrm{CT}$, the $\mathrm{CT}$ must be measured at the same time of the day. However,

Suk Ho Byeon

shbyeon@yuhs.ac

Institute of Vision Research, Department of Ophthalmology, Yonsei University College of Medicine, 50-1 Yonsei-ro, Seodaemun-Gu, Seoul, South Korea 03722 information regarding the time of measurement was not available because of our retrospective study design. A previous prospective study showed that the difference in mean CT did not exceed $10 \mu \mathrm{m}$ from 9:00 AM to 6:00 PM [4]. As our retrospective study showed a relatively large difference in CT between the DRPE and PDR groups (94.68 $113.56 \mu \mathrm{m}$ ), it may be appropriate to assume that CT was larger in the DRPE group than in the PDR group.

Compliance with ethical standards

Conflict of Interest The authors declare that they have no conflict of interest.

\section{References}

1. Kang EC, Seo Y, Byeon SH (2016) Diabetic retinal pigment epitheliopathy: fundus autofluorescence and spectral-domain optical coherence tomography findings. Graefes Arch Clin Exp Ophthalmol. doi:10.1007/s00417-016-3336-8

2. Tan KA, Gupta P, Agarwal A, Chhablani J, Cheng CY, Keane PA, Agrawal R (2016) State of science: Choroidal thickness and systemic health. Surv Ophthalmol. doi:10.1016/j.survophthal.2016.02.007

3. Meng W, Butterworth J, Malecaze F, Calvas P (2011) Axial length of myopia: a review of current research. Ophthalmologica 225:127-134

4. Chakraborty R, Read SA, Collins MJ (2011) Diurnal variations in axial length, choroidal thickness, intraocular pressure, and ocular biometrics. Invest Ophthalmol Vis Sci 52:5121-5129 